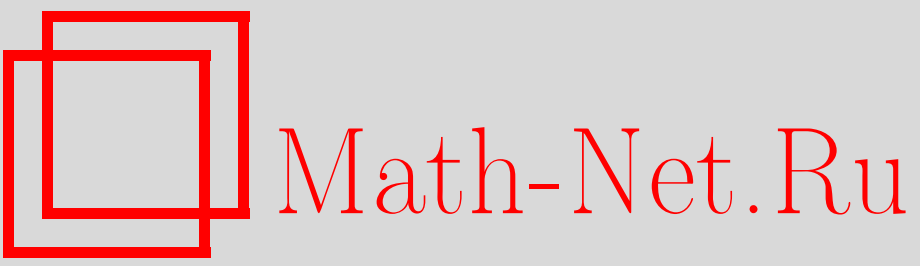

Д. А. Славнов, Необходимые и достаточные постулаты квантовой механики, ТМФ, 2005, том 142, номер 3, 510-529

DOI: https://doi.org/10.4213/tmf1795

Использование Общероссийского математического портала Math-Net.Ru подразумевает, что вы прочитали и согласны с пользовательским соглашением

http://www.mathnet.ru/rus/agreement

Параметры загрузки:

IP: 54.198 .187 .58

26 апреля 2023 г., 13:48:38 
ТЕОРЕТИЧЕСКАЯ

И МАТЕМАТИЧЕСКАЯ

ФИЗИКА

Том 142, № 3

март, 2005

(C) 2005 г.

Д. А. Славнов*

\section{НЕОБХОДИМЫЕ И ДОСТАТОЧНЫЕ ПОСТУЛАТЫ КВАНТОВОЙ МЕХАНИКИ}

Описана система аксиом, которая, с одной стороны, достаточна для построения стандартного математического аппарата квантовой механики, а с другой, необходима с феноменологической точки зрения. В предлагаемой схеме гильбертово пространство и линейные операторы являются лишь вторичными структурами теории. В качестве первичных структур рассматриваются элементы некоммутативной алгебры (наблюдаемые) и функционалы на этой алгебре, которые ассоциируются с результатами единичного наблюдения

Ключевые слова: квантовые постулаты, квантовое измерение, алгебра наблюдаемых, физическая реальность.

\section{1. ВВЕДЕНИЕ}

Базовыми понятиями современной стандартной квантовой механики являются гильбертово пространство и линейные операторы в этом пространстве. Математически четко квантовую механику на основе этих понятий сформулировал фон Нейман [1]. Аппарат гильбертова пространства стал математической основой тех огромных успехов, которых достигла квантовая механика.

Однако такое, казалось бы, безупречное построение теории имеет свои недостатки, что отметил Сигал [2]: “Эти аксиомы просты в техническом отношении, но интуитивно совершенно неясны и представляются возникшими ad hoc".

Другими словами примерно то же самое можно выразить следуюшим образом. Аксиомы стандартной квантовой механики являются феноменологически достаточными, т.е. исходя из них можно описать практически все наблюдаемые эффекты. Однако их феноменологическая необходимость не ясна. Такая ситуация может привести к нежелательным последствиям. Построенная на их основе теория может оказаться "переопределенной”. Косвенным указанием на то, что в стандартной квантовой механике такая угроза существует, являются многочисленные "парадоксы": парадокс Эйнштейна-Подольского-Розена [3], "кот Шредингера" [4], [5] и т.п. Сразу следует оговориться,

* Московский государственный университет, Москва, Россия. E-mail: slavnov@goa.bog.msu.ru 
что отношение к "парадоксам" очень неоднозначное. Наиболее активные приверженцы стандартной квантовой механики полностью отрицают наличие каких-либо парадоксов.

Так или иначе, крайне желательно в качестве постулатов брать такие утверждения, которые в эксперименте могут быть проверены непосредственно. В противном случае, когда проверяются отдаленные последствия, всегда сушествует опасность, что мы проверили не все из них, и среди непроверенных могут оказаться противоречащие эксперименту. В техническом отношении такая система аксиом может оказаться менее эффективной, однако она будет более надежной. В настоящей статье предпринята попытка дать именно такую формулировку основных положений квантовой механики. Предлагаемая система аксиом не является в этом плане идеальной, мы лишь стремились минимизировать техническую составляющую.

Еще одним уязвимым местом подхода, основанного на гильбертовом пространстве, является то, что гильбертово пространство чуждо классической физике. В классической физике используется фазовое пространство, которое, в свою очередь, чуждо стандартной квантовой механике. Философ сказал бы, что классическая и квантовая физики используют разные парадигмы. Этот термин можно понимать как некоторые априорные правила игры. Было бы крайне желательно построить схему, подходяшую как для классической, так и квантовой механики. Соответствуюшая попытка предпринята в данной статье. Это отнюдь не значит, что квантовая механика сводится к классической. Вместе с тем построение ведется в рамках классической парадигмы.

Классическая парадигма - это прежде всего классическая формальная логика и представление о наличии причинно-следственной связи как между физическими явлениями, так и между логическими утверждениями. Далее - предположение о сушествовании физических реальностей, которые являются носителями причин физических явлений, и предположение о том, что вероятностные суждения подчиняются классической колмогоровской теории вероятностей.

Обычно считается, что эти положения несовместимы с математической схемой, принятой в квантовой механике. Здесь предпринята попытка доказать обратное. Эта статья является дальнейшим развитием идей, сформулированных в статьях [6], [7].

\section{2. НАБЛЮДАЕМЫЕ, СОСТОЯНИЯ И ИЗМЕРЕНИЯ}

Начнем с рассмотрения классической физической системы. Базовым понятием для такой системы является "наблюдаемая". Это понятие представляется самоочевидным и не нуждаюшимся в точном определении. Эвристически наблюдаемая - это такой атрибут исследуемой физической системы, для которого с помощью измерения можно получить некоторое численное значение. В дальнейшем будет предполагаться, что измерение идеально, т.е. выполняется с идеальной точностью.

Основным свойством наблюдаемых является то, что их можно умножать на действительные числа, складывать и перемножать между собой. Иначе говоря, они образуют действительную алгебру $\mathfrak{A}_{\mathrm{cl}}$. Задание физической системы предполагает установление соотношений между различными наблюдаемыми, т.е. фиксирование алгебры $\mathfrak{A}_{\mathrm{cl}}$. 
В классическом случае алгебра оказывается коммутативной.

Фиксирование наблюдаемой $\hat{A}$ еше ничего не говорит о значении $A$ этой наблюдаемой, которое будет получено при измерении в конкретной ситуации. Фиксирование значений наблюдаемых реализуется заданием состояния физической системы. В математических терминах этому соответствует задание функционала $\varphi(\hat{A})$ на алгебре $\mathfrak{A}_{\text {сl }}$.

Опыт показывает, что сумме и произведению наблюдаемых соответствует сумма и произведение результатов измерений:

$$
\hat{A}_{1}+\hat{A}_{2} \rightarrow A_{1}+A_{2}, \quad \hat{A}_{1} \hat{A}_{2} \rightarrow A_{1} A_{2}
$$

В связи с этим в дальнейшем будет полезным следуюшее определение [8].

ОПРЕДЕлЕНИЕ 1. Пусть $\mathfrak{B}$ - действительная коммутативная алгебра и $\tilde{\varphi}-$ линейный функционал на $\mathfrak{B}$. Если $\tilde{\varphi}\left(\widehat{B}_{1} \widehat{B}_{2}\right)=\tilde{\varphi}\left(\widehat{B}_{1}\right) \tilde{\varphi}\left(\widehat{B}_{2}\right)$ для всех $\widehat{B}_{1} \in \mathfrak{B}$ и $\widehat{B}_{2} \in \mathfrak{B}$, то функционал $\tilde{\varphi}$ называется действительным гомоморфизмом на алгебре $\mathfrak{B}$.

Используя это определение, мы можем сказать, что состояние - действительньй гомоморфизм на алгебре наблюдаемых. Физически состояние - это некоторьй атрибут физической системы, вычлененной из окружающей среды. Система может быть как изолированной, так и неизолированной. В последнем случае считается, что внешнее влияние сводится к воздействию внешних полей. Предполагается, что состояние определяется некоторой локальной реальностью, которая включает в себя внутреннюю структуру физической системы, а также структуру внешнего поля в области локализации системы.

В процессе измерения физическая система подвергается воздействию со стороны измерительного прибора. По характеру этого воздействия измерения можно подразделить на два типа: воспроизводимые и невоспроизводимые. Воспроизводимое измерение характеризуется тем, что, несмотря на возмушение, которое испытывает система при каждом измерении, многократное измерение одной наблюдаемой дает один и тот же результат. Предполагается, что в интервале между измерениями система изолирована от внешнего воздействия, а возможное изменение наблюдаемой при свободной эволюции мы умеем учитывать. В дальнейшем нас в основном будут интересовать воспроизводимые измерения. Поэтому в термин “измерение" мы будем включать, если специально не оговорено, требование воспроизводимости.

Особый интерес представляет проблема воспроизводимости, когда мы проводим измерения нескольких наблюдаемых для одной физической системы. Пусть мы, например, измеряем наблюдаемую $\hat{A}$, затем наблюдаемую $\widehat{B}$, потом опять наблюдаемую $\hat{A}$ и, наконец, наблюдаемую $\widehat{B}$. Если результаты повторных измерений для каждой наблюдаемой будут совпадать с результатами первичных измерений, то такие измерения назовем совместимыми. Если для наблюдаемых $\hat{A}$ и $\widehat{B}$ сушествуют приборы, которые позволяют осуществить совместимые измерения, то такие наблюдаемые назовем совместимыми, или одновременно измеримыми.

Опыт показывает, что у классических физических систем все наблюдаемые совместимы. В противоположность этому в квантовом случае сушествуют как совместимые, так и несовместимые наблюдаемые. 
В стандартной квантовой механике этот факт квалифицируется как “принцип дополнительности". Мы будем рассматривать его просто как свидетельство того, что для измерения двух несовместимых наблюдаемых требуются приборы, которые исключают друг друга.

Хотя в квантовом случае наблюдаемые также обладают алгебраическими свойствами, построить из них замкнутую алгебру, которая была бы действительна, коммутативна и ассоциативна, не удается. С другой стороны, с технической точки зрения крайне желательно иметь возможность использовать продвинутьй математический аппарат теории алгебр. Поэтому мы примем такой компромиссный вариант первого постулата.

ПостулАт 1. Наблюдаемые величины образуют множество $\mathfrak{A}_{+}$эрмитовых элементов инволютивной ассоциативной (в обшем случае) некоммутативной алгебры $\mathfrak{A}$, удовлетворяющей следующим условиям: для каждого элемента $\widehat{R} \in \mathfrak{A}$ найдется эрмитов элемент $\hat{A}\left(\hat{A}^{*}=\hat{A}\right)$ такой, что $\widehat{R}^{*} \widehat{R}=\hat{A}^{2}$; если $\widehat{R}^{*} \widehat{R}=0$, то $\widehat{R}=0$.

Предполагается, что в алгебре имеется единичный элемент $\hat{I}$. Элементы алгебры $\mathfrak{A}$ будем называть динамическими величинами.

Постулат 1 не лишен полностью технической составляюшей, но она сушественно меньше, чем в соответствующем постулате стандартной квантовой механики. Там дополнительно постулируется, что динамические величины являются линейными операторами в гильбертовом пространстве. Этот факт вряд ли можно признать самоочевидным.

Непосредственно из квантовых измерений следует

ПостулАт 2. Совместимым (одновременно измеримым) наблюдаемым соответствуют взаимно коммутируюшие элементы множества $\mathfrak{A}_{+}$.

В связи с этим постулатом в дальнейшем особо важную роль будут играть действительные коммутативные подалгебры алгебры $\mathfrak{A}$

Обозначим через $\mathfrak{Q}\left(\mathfrak{Q} \equiv\{\widehat{Q}\} \in \mathfrak{A}_{+}\right)$максимальную действительную коммутативную подалгебру алгебры $\mathfrak{A}$. Это подалгебра совместимых наблюдаемых. Если алгебра $\mathfrak{A}$ коммутативна (алгебра классических динамических величин), то такая подалгебра единственна. Если алгебра $\mathfrak{A}$ некоммутативна (алгебра квантовых динамических величин), то существует много таких разных подалгебр $\mathfrak{Q}_{\xi}(\xi \in \Xi)$. Более того, множество $\Xi$ имеет мошность континуума. В самом деле, даже если алгебра $\mathfrak{A}$ имеет две некоммутативные эрмитовы образующие $\hat{A}_{1}$ и $\hat{A}_{2}$, то любая действительная алгебра $\mathfrak{Q}_{\alpha}$ с образуюшей $\widehat{B}(\alpha)=\hat{A}_{1} \cos \alpha+\hat{A}_{2} \sin \alpha$ будет алгеброй типа $\mathfrak{Q}$.

Так же как для классической системы, в квантовом случае значение наблюдаемой выявляется в эксперименте. Иными словами, результат измерения описывается некоторым функционалом, областью определения которого является множество $\mathfrak{A}_{+} \cdot$ Однако структура этого функционала оказывается значительно более сложной, чем в классическом случае.

Из квантовой физической системы, описываемой алгеброй $\mathfrak{A}$, мы можем вычленить подсистему, описываемую подалгеброй $\mathfrak{Q}_{\xi}$ (соответствующими степенями свободы). Так как подалгебра $\mathfrak{Q}_{\xi}$ коммутативна, то вычлененная подсистема может рассматриваться как классическая. Ее состояние можно описать с помошью функционала $\varphi_{\xi}$, ко-

4 Теоретическая и математическая физика, т. 142, № 3, 2005 г. 
торый является действительным гомоморфизмом на алгебре $\mathfrak{Q}_{\xi}$. Конечно, эта классическая подсистема не изолирована от остальной части квантовой системы, но изолированность не является обязательным условием вычленения классической подсистемы. Состояние классической системы определяется некоторой локальной физической реальностью; было бы странным, если то же самое не было бы справедливо и для классической подсистемы.

ОПРЕДЕЛЕНИЕ 2. Назовем многослойным функционалом $\varphi=\left\{\varphi_{\xi}\right\}$ совокупность функционалов $\varphi_{\xi}$, в которой $\xi$ пробегает множество $\Xi$, а областями определения функционалов $\varphi_{\xi}$ являются подалгебры $\mathfrak{Q}_{\xi}$.

В квантовом измерении в каждом индивидуальном эксперименте мы имеем дело только с наблюдаемыми, принадлежашими какой-нибудь одной подалгебре $\mathfrak{Q}_{\xi}$. Результат такого измерения определяется функционалом $\varphi_{\xi}$. Задание функционала $\varphi$ фиксирует все такие функционалы. Поэтому мы можем объявить $\varphi$ состоянием квантовой системы. Чтобы не путать с термином, используемым в стандартной квантовой механике, будем называть $\varphi$ физическим состоянием.

В качестве итога сформулируем следующий постулат.

ПостулАт 3. Результат каждого индивидуального эксперимента по измерению наблюдаемых физической системы определяется физическим состоянием этой системы. Физическое состояние описывается многослойным функционалом $\varphi=\left\{\varphi_{\xi}\right\}, \xi \in \Xi$, заданным на $\mathfrak{A}_{+}$, для которого сужение $\varphi_{\xi}(\hat{A})$ на каждую подалгебру $\mathfrak{Q}_{\xi}$ является действительным гомоморфизмом на алгебре $\mathfrak{Q}_{\xi}$.

Обратим внимание, что никаких дополнительных предположений о свойствах функционалов $\varphi_{\xi}$ не делается. В частности, не предполагается, что

$$
\varphi_{\xi}(\hat{A})=\varphi_{\xi^{\prime}}(\hat{A})
$$

если $\hat{A} \in \mathfrak{Q}_{\xi} \cap \mathfrak{Q}_{\xi^{\prime}}$. Конечно, для некоторых функционалов $\varphi$ равенство (1) может выполняться. Будем говорить, что функционал $\varphi$ стабилен на наблюдаемой $\hat{A}$, если равенство (1) выполняется для всех допустимых $\xi$ и $\xi^{\prime}$.

Равенство (1) кажется само собой разумеющимся. Поэтому возможность его нарушения требует специального комментария.

Измерение - это результат взаимодействия двух систем: исследуемой физической системы (классической или квантовой) и классического измерительного прибора. Априорно результат может зависеть как от исследуемой системы, так и от прибора. Зависимость от прибора является паразитной. Чтобы ее исключить, используют калибровку прибора. Сушность ее заключается в следующем. Берут пробную физическую систему и подвергают ее воспроизводимому измерению. Затем повторно измеряют ее прибором, который хотят прокалибровать. Результаты обоих измерений должны совпадать (в пределах допустимой погрешности). Только прибор, который многократно выдерживает такое испытание, заслуживает названия измерительного прибора. 
Так можно исключить зависимость результата измерения от микросостояния прибора. Однако предположим, что каждый измерительный прибор в силу своих конструктивных особенностей, ориентации в пространстве и каких-то других макроскопических свойств относится к одному из типов, который может быть помечен параметром $\xi$ $(\xi \in \Xi)$. Будем считать, что следующие свойства определяют принадлежность прибора к типу $\xi$. Во-первых, прибор предназначен для измерения наблюдаемой (наблюдаемых) из подалгебры $\mathfrak{Q}_{\xi}$. Во-вторых, при измерении наблюдаемой $\hat{A} \in \mathfrak{Q}_{\xi}$ для системы, находяшейся в физическом состоянии $\varphi$, результат будет $A_{\xi}=\varphi_{\xi}(\hat{A})$.

Оказьвается, что исключить зависимость от параметра $\xi$ нельзя. Для доказательства этого утверждения достаточно привести хотя бы один пример такого сценария взаимодействия измерительного прибора с исследуемым объектом, в котором зависимость от $\xi$ никакой калибровкой не исключается.

Построим такой пример. Пусть прибор осушествляет воспроизводимое измерение, и его воздействие на физическое состояние исследуемого объекта обладает следующими свойствами: функционал $\varphi_{\xi}$ остается неизменным; многослойный функционал $\varphi$ становится стабильным на наблюдаемой $\hat{A}$; если $\widehat{B} \in \mathfrak{Q}_{\xi}$ и $\varphi$ стабилен на $\widehat{B}$, то он таким и остается. В остальном $\varphi$ изменяется неконтролируемым образом. Элементарно проверяется, что приборы, обладаюшие такими свойствами, будут совместимыми. С другой стороны, эти свойства являются необходимыми для совместимости приборов.

Пусть $\hat{A} \in \mathfrak{Q}_{\xi} \cap \mathfrak{Q}_{\xi^{\prime}}\left(\xi \neq \xi^{\prime}\right)$. Покажем, что для приборов типов $\xi$ и $\xi^{\prime}$ никакой калибровкой нельзя исключить возможность нарушения равенства (1). Чтобы проверить равенство (1), мы должны провести для одной физической системы два измерения: сначала прибором типа $\xi$, затем типа $\xi^{\prime}$, или наоборот. Абсолютно одновременно двумя разными приборами произвести измерения невозможно.

Пусть сначала используется прибор типа $\xi$. В результате мы получим показание $A_{\xi}=\varphi_{\xi}(\hat{A})$, а функционал $\varphi$ преобразуется в функционал $\varphi^{\prime}$. По сценарию этот функционал должен обладать свойством $\varphi_{\xi^{\prime}}^{\prime}(\hat{A})=\varphi_{\xi}^{\prime}(\hat{A})=\varphi_{\xi}(\hat{A})$. Поэтому в результате второго измерения (прибором типа $\xi^{\prime}$ ) мы опять получим $A_{\xi}$.

Если сначала используем прибор типа $\xi^{\prime}$, то в результате тех же действий получим ответ $A_{\xi^{\prime}}$. Однако выяснить, совпадают ли значения $A_{\xi}$ и $A_{\xi^{\prime}}$, невозможно, так как в любом варианте последовательных измерений мы будем получать только один ответ.

Надо подчеркнуть, что классификация приборов по типам $\xi$ - это классификация по характеру взаимодействия прибора и исследуемой системы. Поэтому она определяется не только свойствами прибора, но и исследуемой системой (алгеброй $\mathfrak{A}$ ). Кроме того, даже если прибор взаимодействует только с изолированной частью системы, он может повлиять на значения наблюдаемых, глобальных для всей системы.

Зависимость результата измерения от типа прибора можно рассматривать как реализацию и конкретизацию представлений Бора о зависимости результата от обшего контекста эксперимента. Вместе с тем предлагаемый здесь вариант зависимости не противоречит ни принципу причинности, ни представлению о существовании локальной реальности. Однако локальная реальность - это не определенное значение каждой наблюдаемой для рассматриваемой физической системы, а реакция определенного типа 
измерительного прибора на физическое состояние системы. Только в случае, когда соответствующее физическое состояние стабильно на некоторой наблюдаемой, можно говорить об определенном значении этой наблюдаемой. Коммутативная алгебра $\mathfrak{A}$ имеет только одну максимальную действительную коммутативную подалгебру. Поэтому в классическом случае все измерительные приборы относятся к одному типу, и все физические состояния стабильны на всех наблюдаемых.

В свете сказанного следует по-новому взглянуть на результат, полученный в статье Кочена и Шпеккера [9], в которой доказана запрешающая (no go) теорема. Смысл этой теоремы заключается в том, что для частицы со спином 1 не существует такой внутренней характеристики (физической реальности [3]), которая однозначно предопределяет значения квадратов проекций спина на три взаимно ортогональные направления.

В описываемом в данной статье подходе условия теоремы Кочена-Шпеккера не выполняются. Действительно, используемые в статье [9] наблюдаемые $\left(\widehat{S}_{x}^{2}, \widehat{S}_{y}^{2}, \widehat{S}_{z}^{2}\right)$ являются совместимыми. Совместимыми являются также наблюдаемые $\left(\widehat{S}_{x}^{2}, \widehat{S}_{y^{\prime}}^{2}, \widehat{S}_{z^{\prime}}^{2}\right)$, где направления $x, y^{\prime}, z^{\prime}$ также ортогональны между собой, но направления $y, z$ не параллельны направлениям $y^{\prime}, z^{\prime}$. Наблюдаемые $\left(\widehat{S}_{y}^{2}, \widehat{S}_{z}^{2}\right)$ не совместимы с наблюдаемыми $\left(\widehat{S}_{y^{\prime}}^{2}, \widehat{S}_{z^{\prime}}^{2}\right)$. Приборы, согласованные с наблюдаемыми $\left(\widehat{S}_{x}^{2}, \widehat{S}_{y}^{2}, \widehat{S}_{z}^{2}\right)$ и $\left(\widehat{S}_{x}^{2}, \widehat{S}_{y^{\prime}}^{2}, \widehat{S}_{z^{\prime}}^{2}\right)$, относятся к разным типам. Поэтому эти приборы не обязательно должны давать один и тот же результат при измерении квадрата проекции спина на направление $x$; в теореме Кочена-Шпеккера это неявно предполагается.

Можно показать [8], что функционалы $\varphi_{\xi}(\hat{A})$ обладают следующими свойствами:

$$
\begin{array}{r}
\varphi_{\xi}(0)=0 \\
\varphi_{\xi}(\hat{I})=1 ; \\
\varphi_{\xi}\left(\hat{A}^{2}\right) \geqslant 0 ; \\
\lambda \in \sigma\left(\hat{A} ; \mathfrak{Q}_{\xi}\right),
\end{array}
$$

если $\lambda=\varphi_{\xi}(\hat{A})$;

$$
\lambda=\varphi_{\xi}(\hat{A})
$$

для некоторого $\varphi_{\xi}(\hat{A})$, если $\lambda \in \sigma\left(\hat{A} ; \mathfrak{Q}_{\xi}\right)$. Здесь $\sigma\left(\hat{A} ; \mathfrak{Q}_{\xi}\right)$ - спектр элемента $\hat{A}$ в подалгебре $\mathfrak{Q}_{\xi}$. Так как подалгебра $\mathfrak{Q}_{\xi}$ максимальна, то в алгебре $\mathfrak{A}$ спектр будет таким же (см. например, [8]). Вспомним, что по определению $\lambda \in \sigma(\hat{A} ; \mathfrak{A})$, если и только если элемент $\hat{A}-\lambda \hat{I}$ не имеет обратного в алгебре $\mathfrak{A}$.

В стандартной квантовой механике, чтобы учесть свойства измерений, описываемые соотношениями (2), приходится вводить специальньй постулат. Соотношения (2) имеют большой конструктивный потенциал. В частности, свойства (2г) и (2д) позволяют построить все множество физических состояний, допустимых для рассматриваемой физической системы.

Ясно, что для построения многослойного функционала $\varphi$ необходимо и достаточно построить все его составляюшие $\varphi_{\xi}$. Каждый функционал $\varphi_{\xi}$ можно построить следуюшим образом. В подалгебре $\mathfrak{Q}_{\xi}$ произвольным образом выбираем систему $G\left(\mathfrak{Q}_{\xi}\right)$ 
независимых образующих. Строим $\varphi_{\xi}$ как некоторое отображение $G\left(\mathfrak{Q}_{\xi}\right)$ на множество точек спектров соответствующих элементов. На остальных элементах подалгебры $\mathfrak{Q}_{\xi}$ функционал $\varphi_{\xi}$ определяется своими свойствами линейности и мультипликативности.

Процедура построения функционалов $\varphi_{\xi}$ заведомо непротиворечива, если для разных $\xi$ функционалы строятся независимо друг от друга. Конечно, при этом равенство (1) может нарушаться.

Однако всегда можно построить многослойный функционал $\varphi$, стабильный на всех наблюдаемых, принадлежащих любой одной из подалгебр $\mathfrak{Q}_{\xi}$. Для этого достаточно начать построение функционала $\varphi$ именно с этой подалгебры, используя только что описанную процедуру. На другой подалгебре $\mathfrak{Q}_{\xi^{\prime}}$ функционал $\varphi_{\xi^{\prime}}$ строим следующим образом. Пусть $\mathfrak{Q}_{\xi} \cap \mathfrak{Q}_{\xi^{\prime}}=\mathfrak{Q}_{\xi \xi^{\prime}}$ и $G\left(\mathfrak{Q}_{\xi \xi^{\prime}}\right)$ - независимые образующие подалгебры $\mathfrak{Q}_{\xi \xi^{\prime}}$. Пусть $\bar{G}\left(\mathfrak{Q}_{\xi \xi^{\prime}}\right)$ - дополнение этих образующих до множества образующих подалгебры $\mathfrak{Q}_{\xi^{\prime}}$. Если $\hat{A} \in \mathfrak{Q}_{\xi \xi^{\prime}}$, то полагаем $\varphi_{\xi^{\prime}}(\hat{A})=\varphi_{\xi}(\hat{A})$. Если $\hat{A} \in \bar{G}\left(\mathfrak{Q}_{\xi \xi^{\prime}}\right)$, то строим $\varphi_{\xi^{\prime}}(\hat{A})$ как отображение элемента $\hat{A}$ в одну из точек его спектра. На остальных элементах подалгебры $\mathfrak{Q}_{\xi^{\prime}}$ функционал $\varphi_{\xi^{\prime}}$ строится по линейности и мультипликативности.

В квантовом измерении физическое состояние не может быть зафиксировано однозначно. Действительно, так как приборы, предназначенные для измерения несовместимых наблюдаемых, несовместимы, то в одном эксперименте мы можем измерить наблюдаемые, принадлежашие одной максимальной коммутативной подалгебре $\mathfrak{Q}_{\xi}$. В результате мы установим только значения функционала $\varphi_{\xi}$. В остальном многослойный функционал $\varphi$ останется неопределенным. Повторное измерение с использованием другого типа прибора даст новую информацию, но неконтролируемым образом возмутит физическое состояние, возникшее после первого измерения. Поэтому информация, полученная в первом измерении, станет бесполезной.

В связи с этим удобно принять следующее определение.

ОПРЕДЕлЕНИЕ 3. Многослойные функционалы $\varphi$ назовем $\varphi_{\xi}$-эквивалентными, если они имеют одно и то же сужение $\varphi_{\xi}$ на подалгебру $\mathfrak{Q}_{\xi}$.

Таким образом, в квантовом измерении мы можем установить только класс эквивалентности, к которому принадлежит исследуемое физическое состояние. В воспроизводимом измерении наблюдаемых, принадлежащих подалгебре $\mathfrak{Q}_{\xi}$, легко узнать процедуру приготовления состояния в стандартной квантовой механике. Далее это состояние будем называть квантовым состоянием и примем следуюшее определение.

ОПРеДЕЛЕНИЕ 4. Квантовым состоянием $\Psi_{\varphi \xi}(\cdot)$ назовем класс $\{\varphi\}_{\varphi \xi} \varphi_{\xi^{-э к в и в а-~}}$ лентных физических состояний, стабильных на подалгебре $\mathfrak{Q}_{\xi}$.

В действительности такое определение квантового состояния удобно только для систем, в которых нет тождественных частиц. Дело в том, что измерительный прибор не может установить, какая из тож дественных частиц в него попала. Поэтому удобно несколько обобшить понятие эквивалентности. Будем говорить, что функционал $\varphi$ слабо $\varphi_{\xi}$-эквивалентен функционалу $\varphi^{\prime}$, если сужение $\varphi_{\xi}$ функционала $\varphi$ на подалгебру $\mathfrak{Q}_{\xi}$ совпадает с сужением $\varphi_{\xi^{\prime}}^{\prime}$ функционала $\varphi^{\prime}$ на подалгебру $\mathfrak{Q}_{\xi^{\prime}}$. Здесь предполагается, 
что подалгебра $\mathfrak{Q}_{\xi^{\prime}}$ получается из подалгебры $\mathfrak{Q}_{\xi}$ заменой наблюдаемых одной из тождественных частиц на соответствующие наблюдаемые другой.

Для систем с тождественными частицами в определении квантового состояния эквивалентность следует заменить на слабую эквивалентность. Далее будем считать, что, если необходимо, такая замена сделана.

\section{3. КВАНТОВЫЙ АНСАМБЛЬ И ТЕОРИЯ ВЕРОЯТНОСТЕЙ}

В настоящее время наиболее математически развитой является колмогоровская теория вероятностей [10]. Обычно считается, что для квантовых систем требуется специальная квантовая теория вероятностей. В данной статье будет зашишаться мнение, что и в квантовом случае вполне достаточно классической колмогоровской теории вероятностей, нужно только учесть особенность квантовых измерений [7].

В основе колмогоровской теории вероятностей (см., например, [10], [11]) лежит так называемое вероятностное пространство $(\Omega, \mathcal{F}, P)$.

Первая составляющая $\Omega$ - множество (пространство) элементарных событий. Физический смысл элементарных событий специально не оговаривается, но считается, что они являются взаимоисключающими, и в каждом испытании реализуется одно и только одно элементарное событие. В нашем случае в качестве элементарного события будет выступать физическое состояние $\varphi$.

Помимо элементарного события вводится еше понятие "случайное событие" или просто "событие". Каждое событие $F$ отождествляется с некоторым подмножеством множества $\Omega$. Считается, что произошло событие $F$, если реализовалось одно из элементарных событий, принадлежащих этому подмножеству $(\varphi \in F)$. Предполагается, что в каждом испытании мы можем установить, произошло событие или нет. От элементарных событий этого не требуется.

Наборы подмножеств множества $\Omega$ (включающих само множество $\Omega$ и пустое множество $\varnothing)$ наделяются структурой булевых алгебр. Алгебраическими операциями являются пересечения подмножеств, их объединения и дополнения до $\Omega$. Булева алгебра, замкнутая относительно счетного числа объединений и пересечений, называется $\sigma$-алгеброй.

Второй составляюшей вероятностного пространства является некоторая $\sigma$-алгебра $\mathcal{F}$. Множество $\Omega$, в котором выбрана определенная $\sigma$-алгебра $\mathcal{F}$, назьвается измеримым пространством.

Наконец, третья составляюшая вероятностного пространства - вероятностная мера $P$. Это отображение алгебры $\mathcal{F}$ в множество действительных чисел, удовлетворяющее следующим условиям: а) $0 \leqslant P(F) \leqslant 1$ для всех $F \in \mathcal{F}, P(\Omega)=1 ;$ б) $P\left(\sum_{j} F_{j}\right)=$ $\sum_{j} P\left(F_{j}\right)$ для любой счетной совокупности непересекаюшихся подмножеств $F_{j} \in \mathcal{F}$.

Обратим внимание, что вероятностная мера определена только для событий, входяших в алгебру $\mathcal{F}$. Для элементарных событий вероятность, вообше говоря, не определена.

Рассмотрим теперь приложение основных принципов теории вероятностей к проблеме квантовых измерений. Основной целью квантового эксперимента является нахож- 
дение вероятностных распределений тех или иных наблюдаемых величин. При использовании определенной измерительной аппаратуры мы можем получить такое распределение для некоторой совокупности совместимых наблюдаемых. С точки зрения теории вероятностей, выбирая определенную измерительную аппаратуру, мы выбираем определенную $\sigma$-алгебру $\mathcal{F}$.

Предположим, мы проводим типичный квантовый эксперимент. У нас имеется ансамбль квантовых систем, находящихся в определенном квантовом состоянии. Например, частицы со спином $1 / 2$ с проекцией спина на ось $x$, равной $1 / 2$. Пусть мы хотим исследовать распределение проекций спина на направления, составляюшие с осью $x$ углы $\theta_{1}$ и $\theta_{2}$. Соответствуюшие наблюдения несовместимы, в одном эксперименте обе наблюдаемые мы измерить не можем. Поэтому мы должны провести две групшы экспериментов с использованием разной измерительной аппаратуры. В нашем конкретном случае магниты в приборе Штерна-Герлаха должны быть различным образом ориентированы в пространстве.

Одну группу экспериментов мы можем описать с помощью вероятностного пространства $\left(\Omega, \mathcal{F}_{1}, P_{1}\right)$, другую - с помошью $\left(\Omega, \mathcal{F}_{2}, P_{2}\right)$. Хотя в обоих случаях пространство элементарных событий $\Omega$ одно и то же, вероятностные пространства разные. Для придания этим пространствам свойства измеримости в них введены разные $\sigma$-алгебры $\mathcal{F}_{1}$ и $\mathcal{F}_{2}$.

Формально, чисто математически [11], мы можем построить $\sigma$-алгебру $\mathcal{F}_{12}$, которая включает в себя как алгебру $\mathcal{F}_{1}$, так и алгебру $\mathcal{F}_{2}$. Такая алгебра называется порожденной алгебрами $\mathcal{F}_{1}$ и $\mathcal{F}_{2}$. Она содержит, помимо подмножеств $F_{i}^{(1)} \in \mathcal{F}_{1}$ и $F_{j}^{(2)} \in \mathcal{F}_{2}$ множества $\Omega$, также всевозможные пересечения и объединения этих подмножеств. Однако с физической точки зрения такая $\sigma$-алгебра будет неприемлемой.

Действительно, событие $F_{i j}=F_{i}^{(1)} \cap F_{j}^{(2)}$ состоит в том, что для одного квантового объекта значения двух несовместимых наблюдаемых лежат в строго определенной области. Для квантовой системы принципиально невозможно поставить эксперимент, который смог бы выделить такое событие. Поэтому для такого события вообще не существует понятия “вероятность", т.е. подмножеству $F_{i j}$ не соответствует никакая вероятностная мера, и $\sigma$-алгебра $\mathcal{F}_{12}$ не годится для построения вероятностного пространства. Здесь проявляется принципиальная особенность приложения теории вероятностей к квантовым системам: не всякая математически возможная $\sigma$-алгебра является физически допустимой.

Таким образом, элементу измеримого пространства $(\Omega, \mathcal{F})$ в эксперименте соответствует пара - квантовый объект (например, находящийся в определенном квантовом состоянии) плюс определенный тип измерительной аппаратуры, позволяющий зафиксировать событие определенного вида. Каждый такой прибор может выделить события, соответствующие некоторому набору совместимых наблюдаемых величин, т.е. принадлежащих одной подалгебре $\mathfrak{Q}_{\xi}$. Если мы считаем, что каждый измерительный прибор относится к некоторому типу $\xi$, то $\sigma$-алгебра $\mathcal{F}$ будет зависеть от параметра $\xi: \mathcal{F}=\mathcal{F}_{\xi}$.

Особенность квантового эксперимента требует внимательности при определении одного из основных понятий теории вероятностей - действительной случайной величины. 
Обычно действительная случайная величина определяется как отображение пространства $\Omega$ элементарных событий в расширенную действительную прямую $\bar{R}=$ $[-\infty,+\infty]$. Однако такое определение не учитывает особенностей квантового эксперимента, в котором результат может зависеть от типа измерительного прибора. Поэтому мы примем такое определение.

ОПРЕДЕЛЕНИЕ 5. Действительной случайной величиной называется отображение измеримого пространства $\left(\Omega, \mathcal{F}_{\xi}\right)$ элементарных событий в расширенную действительную прямую.

В приложении к наблюдаемой $\hat{A}$ это будет выглядеть так:

$$
\varphi \stackrel{\hat{A}}{\longrightarrow} A_{\xi}(\varphi) \equiv \varphi_{\xi}(\hat{A}) \in \bar{R}
$$

Назовем квантовым ансамблем множество однотипных (описываемых одной алгеброй $\mathfrak{A})$ физических систем, которые находятся в некотором квантовом состоянии. Эксперимент однозначно свидетельствует в пользу того, что такой ансамбль обладает вероятностными свойствами. Поэтому примем следующий постулат.

ПостулАт 4. Квантовый ансамбль может быть оснашен структурой вероятностного пространства.

Рассмотрим ансамбль физических систем, которые находятся в квантовом состоянии $\Psi_{\varphi \eta}(\cdot), \quad \eta \in \Xi$. Соответственно будем рассматривать класс эквивалентности $\{\varphi\}_{\varphi \eta}$ как пространство $\Omega\left(\varphi_{\eta}\right)$ элементарных событий $\varphi$. Пусть в эксперименте измеряется значение наблюдаемой $\hat{A} \in \mathfrak{Q}_{\xi}$ и используется прибор типа $\xi$. Обозначим через $\left(\Omega\left(\varphi_{\eta}\right), \mathcal{F}_{\xi}\right)$ соответствуюшее измеримое пространство. Пусть $P_{\xi}-$ вероятностная мера на этом пространстве, т.е. $P_{\xi}(F)$ - вероятность события $F \in \mathcal{F}_{\xi}$.

Будем считать, что в эксперименте реализуется событие $\tilde{A}$, если зарегистрированное значение наблюдаемой $\hat{A}$ не больше $\tilde{A}$. Вероятность этого события обозначим $P_{\xi}(\tilde{A})=$ $P\left(\varphi: \varphi_{\xi}(\hat{A}) \leqslant \tilde{A}\right)$. Зная вероятности $P_{\xi}(F)$, с помощью соответствующих суммирований и интегрирований мы можем найти вероятность $P_{\xi}(\tilde{A}) ;$ распределение $P_{\xi}(\tilde{A})$ является маргинальньмм для вероятностей $P_{\xi}(F)$ (см., например, [12]).

Наблюдаемая $\hat{A}$ может принадлежать не только подалгебре $\mathfrak{Q}_{\xi}$, но и другой максимальной подалгебре $\mathfrak{Q}_{\xi^{\prime}}$. Поэтому для определения вероятности события $\tilde{A}$ мы можем использовать прибор типа $\xi^{\prime}$. В этом случае для вероятности мы могли бы получить другое значение $P_{\xi^{\prime}}(\tilde{A})$. Однако опыт показывает, что вероятности не зависят от используемого измерительного прибора. Поэтому мы должны принять еще один постулат.

ПостулАт 5. Пусть наблюдаемая $\hat{A} \in \mathfrak{Q}_{\xi} \cap \mathfrak{Q}_{\xi^{\prime}}$, тогда вероятность обнаружить событие $\tilde{A}$ для системы, находяшейся в квантовом состоянии $\Psi_{\varphi \eta}(\cdot)$, не зависит от типа используемого прибора, т.е. $\left.P\left(\varphi: \varphi_{\xi}(\hat{A})\right) \leqslant \tilde{A}\right)=P\left(\varphi: \varphi_{\xi^{\prime}}(\hat{A}) \leqslant \tilde{A}\right)$.

Поэтому, несмотря на то что функционал $\varphi$ многослойный, для вероятности события $\tilde{A}$ мы вправе использовать обозначение $P(\varphi: \varphi(\hat{A}) \leqslant \tilde{A})$. 
Пусть мы имеем ансамбль квантовых систем, находящихся в квантовом состоянии $\Psi_{\varphi \eta}(\cdot)$, и проводим с этим ансамблем серию экспериментов, в которых измеряется наблюдаемая $\hat{A}$. В любой реальной серии мы имеем дело с конечным множеством физических состояний. В идеальной серии это множество может быть счетным. Обозначим через $\{\varphi\}_{\varphi \eta}^{A}$ случайную счетную выборку из пространства $\Omega(\eta)$, которая содержит все физические состояния из реальной серии. По закону больших чисел (см. например, [11]) вероятностная мера $P_{\hat{A}}$ в этой выборке однозначно определяется вероятностями $P(\varphi: \varphi(\hat{A}) \leqslant \tilde{A})$.

Вероятностная мера $P_{\hat{A}}$ определяет среднее значение наблюдаемой $\hat{A}$ в выборке $\{\varphi\}_{\varphi \eta}^{A}$ :

$$
\langle\hat{A}\rangle=\int_{\{\varphi\}_{\varphi_{\xi}}^{A}} P_{\hat{A}}(d \varphi) \varphi(\hat{A}) \equiv \Psi_{\varphi \eta}(\hat{A}) .
$$

Это среднее значение не зависит от конкретной выборки, а полностью определяется квантовым состоянием $\Psi_{\varphi \eta}(\cdot)$. Формула (3) определяет функционал (квантовое среднее) на множестве $\mathfrak{A}_{+}$. Этот функционал также будем обозначать символом $\Psi_{\varphi \eta}(\cdot)$. Вся совокупность квантовых экспериментов указывает на то, что мы должны принять следуюший постулат.

Постул ат 6. Функционал $\Psi_{\varphi \eta}(\cdot)$ линеен на множестве $\mathfrak{A}_{+}$.

Это значит, что $\Psi_{\varphi \eta}(\hat{A}+\widehat{B})=\Psi_{\varphi \eta}(\hat{A})+\Psi_{\varphi \eta}(\widehat{B})$ и в том случае, когда $[\hat{A}, \widehat{B}] \neq 0$.

Любой элемент $\widehat{R}$ алгебры $\mathfrak{A}$ однозначно представляется в виде $\widehat{R}=\hat{A}+i \widehat{B}$, где $\hat{A}, \widehat{B} \in \mathfrak{A}_{+}$. Поэтому функционал $\Psi_{\varphi \eta}(\cdot)$ можно однозначно расширить до линейного функционала на алгебре $\mathfrak{A}: \Psi_{\varphi \eta}(\widehat{R})=\Psi_{\varphi \eta}(\hat{A})+i \Psi_{\varphi \eta}(\widehat{B})$.

Определим полунорму элемента $\widehat{R}$ равенством

$$
\|\widehat{R}\|^{2}=\sup _{\varphi_{\xi}} \varphi_{\xi}\left(\widehat{R}^{*} \widehat{R}\right)=\rho\left(\widehat{R}^{*} \widehat{R}\right)
$$

где $\rho\left(\widehat{R}^{*} \widehat{R}\right)$ - спектральный радиус элемента $\widehat{R}^{*} \widehat{R}$ в алгебре $\mathfrak{A}$.

Такое определение допустимо. Во-первых, $\|\widehat{R}\|^{2} \geqslant 0$ благодаря свойству (2в). Далее, в силу определения вероятностной меры

$$
\Psi_{\varphi \eta}\left(\widehat{R}^{*} \widehat{R}\right)=\int_{\{\varphi\}_{\varphi \eta}^{R^{*} R}} P_{\widehat{R}^{*} \widehat{R}}(d \varphi)\left[\widehat{R}^{*} \widehat{R}\right](\varphi) \leqslant \sup _{\varphi_{\xi}} \varphi_{\xi}\left(\widehat{R}^{*} \widehat{R}\right)=\rho\left(\widehat{R}^{*} \widehat{R}\right) .
$$

Выберем $\eta \in \Xi$ такое, что $\widehat{R}^{*} \widehat{R} \in \mathfrak{Q}_{\eta}$. Тогда $\Psi_{\varphi \eta}\left(\widehat{R}^{*} \widehat{R}\right)=\varphi_{\eta}\left(\widehat{R}^{*} \widehat{R}\right)$. Для таких $\eta$

$$
\sup _{\varphi_{\eta}} \Psi_{\varphi \eta}\left(\widehat{R}^{*} \widehat{R}\right)=\sup _{\varphi_{\eta}} \varphi_{\eta}\left(\widehat{R}^{*} \widehat{R}\right)=\rho_{\eta}\left(\widehat{R}^{*} \widehat{R}\right)
$$

где $\rho_{\eta}\left(\widehat{R}^{*} \widehat{R}\right)$ - спектральный радиус в $\mathfrak{Q}_{\eta}$. Так как подалгебра $\mathfrak{Q}_{\eta}$ максимальна, то $\rho_{\eta}\left(\widehat{R}^{*} \widehat{R}\right)=\rho\left(\widehat{R}^{*} \widehat{R}\right)$. Отсюда, учитывая равенства $(4)-(6)$, получаем

$$
\|\widehat{R}\|^{2}=\sup _{\varphi_{\xi}} \varphi_{\xi}\left(\widehat{R}^{*} \widehat{R}\right)=\sup _{\varphi_{\xi}} \Psi_{\varphi \xi}\left(\widehat{R}^{*} \widehat{R}\right)
$$


Так как $\Psi_{\varphi \xi}(\cdot)$ - линейный положительный функционал, то справедливо неравенство Коши-Буняковского-Шварца:

$$
\left|\Psi_{\varphi \xi}\left(\widehat{R}^{*} \widehat{S}\right) \Psi_{\varphi \xi}\left(\widehat{S}^{*} \widehat{R}\right)\right| \leqslant \Psi_{\varphi \xi}\left(\widehat{R}^{*} \widehat{R}\right) \Psi_{\varphi \xi}\left(\widehat{S}^{*} \widehat{S}\right) .
$$

Отсюда следует, что для $\|\widehat{R}\|$ выполняются аксиомы полунормы элемента $\widehat{R}$ (см. например, [13]):

$$
\|\widehat{R}+\widehat{S}\| \leqslant\|\widehat{R}\|+\|\widehat{S}\|, \quad\|\lambda \widehat{R}\|=|\lambda|\|\widehat{R}\|, \quad\left\|\widehat{R}^{*}\right\|=\|\widehat{R}\| .
$$

Рассмотрим теперь множество $J$ элементов $\widehat{R}$ алгебры $\mathfrak{A}$, для которых $\|\widehat{R}\|^{2}=0$. Из неравенства (8) следует, что $J$ является двусторонним идеалом $\mathfrak{A}$. Поэтому можно образовать фактор-алгебру $\mathfrak{A}^{\prime}=\mathfrak{A} / J$. В алгебре $\mathfrak{A}^{\prime}$ из $\|\widehat{R}\|^{2}=0$ следует $\widehat{R}=0$. Поэтому в алгебре $\mathfrak{A}^{\prime}$ равенство (4) будет определять не полунорму, а норму. С другой стороны, можно убедиться, что алгебра $\mathfrak{A}^{\prime}$ несет ту же физическую информацию, что и $\mathfrak{A}$.

Для этой цели рассмотрим две наблюдаемые $\hat{A}$ и $\widehat{B}$, которые одновременно либо принадлежат, либо не принадлежат каждой из подалгебр $\mathfrak{Q}_{\xi}$. Пусть $\hat{A}$ и $\widehat{B}$ удовлетворяют дополнительному условию $\|\hat{A}-\widehat{B}\|=0$. Тогда из равенства (4) следует, что

$$
\varphi_{\xi}(\hat{A})=\varphi_{\xi}(\widehat{B})
$$

для всех $\mathfrak{Q}_{\xi}$, содержаших эти наблюдаемые. Равенство (9) означает, что никакой эксперимент не может различить эти наблюдаемые, поэтому с феноменологической точки зрения их можно отождествить. Переход от алгебры $\mathfrak{A}$ к алгебре $\mathfrak{A}^{\prime}$ позволяет математически реализовать такое отождествление. Чтобы сразу иметь дело с алгеброй типа $\mathfrak{A}^{\prime}$, нужно принять следуюший постулат.

Постулат 7. Если $\sup _{\varphi_{\xi}}\left\|\varphi_{\xi}(\hat{A}-\widehat{B})\right\|=0$, то $\hat{A}=\widehat{B}$.

Постулат 7 носит технический характер. Вместе с тем с точки зрения феноменологии он не накладывает никаких дополнительных ограничений, только упрошает математическое описание физических систем. Далее будем считать, что требование постулата 7 удовлетворяется, и поэтому равенство (7) определяет норму элемента $\widehat{R}$.

Из мультипликативных свойств функционала $\varphi_{\xi}$ следует, что $\varphi_{\xi}\left(\left[\widehat{R}^{*} \widehat{R}\right]^{2}\right)=$ $\left[\varphi_{\xi}\left(\widehat{R}^{*} \widehat{R}\right)\right]^{2}$. Это значит, что $\left\|\widehat{R}^{*} \widehat{R}\right\|=\|\widehat{R}\|^{2}$. Поэтому, если мы пополним алгебру $\mathfrak{A}$ по норме $\|\cdot\|$, то $\mathfrak{A}$ превратится в $C^{*}$-алгебру [14]. Таким образом, алгебра квантовых динамических величин может быть оснашена структурой $C^{*}$-алгебры.

В стандартном алгебраическом подходе к квантовой теории это утверждение принимается в качестве исходной аксиомы. Математически это, конечно, очень удобно. Однако с феноменологической точки зрения необходимость такой аксиомы остается совершенно неясной.

\section{4. $C^{*}$-АЛГЕБРА И ГИЛЬБЕРТОВО ПРОСТРАНСТВО}

Замечательной особенностью $C^{*}$-алгебры является то, что любая $C^{*}$-алгебра изометрически изоморфна подалгебре линейных ограниченных операторов в подходяшем 
гильбертовом пространстве [13], [14]. Это позволит нам в дальнейшем использовать привычный аппарат гильбертова пространства.

Процедурой, которая реализует связь $C^{*}$-алгебры с гильбертовым пространством, является так называемая каноническая конструкция Гельфанда-Наймарка-Сигала (ГНС) (см., например, [13]). Вкратце она состоит в следуюшем.

Пусть имеется некоторая $C^{*}$-алгебра $\mathfrak{A}$ и линейньй положительный функционал $\Psi_{0}$ на этой алгебре. Будем считать два элемента $\widehat{R}, \widehat{R}^{\prime} \in \mathfrak{A}$ эквивалентными, если для любого $\widehat{K} \in \mathfrak{A}$ справедливо равенство $\Psi_{0}\left(\widehat{K}^{*}\left(\widehat{R}-\widehat{R}^{\prime}\right)\right)=0$. Обозначим через $\Phi(\widehat{R})$ класс эквивалентности элемента $\widehat{R}$ и рассмотрим множество $\mathfrak{A}\left(\Psi_{0}\right)$ всех классов эквивалентности в $\mathfrak{A}$. Превратим множество $\mathfrak{A}\left(\Psi_{0}\right)$ в линейное пространство, положив $a \Phi(\widehat{R})+b \Phi(\widehat{S})=\Phi(a \widehat{R}+b \widehat{S})$. Определим в $\mathfrak{A}\left(\Psi_{0}\right)$ скалярное произведение формулой

$$
(\Phi(\widehat{R}), \Phi(\widehat{S}))=\Psi_{0}\left(\widehat{R}^{*} \widehat{S}\right) .
$$

Это скалярное произведение порождает в $\mathfrak{A}\left(\Psi_{0}\right)$ норму $\|\Phi(\widehat{R})\|^{2}=\Psi_{0}\left(\widehat{R}^{*} \widehat{R}\right)$. Пополнение по этой норме превращает $\mathfrak{A}\left(\Psi_{0}\right)$ в гильбертово пространство. Каждый элемент $\widehat{S}$ алгебры $\mathfrak{A}$ однозначно представляется в этом пространстве линейным оператором $\Pi(\widehat{S})$, действуюшим по правилу

$$
\Pi(\widehat{S}) \Phi(\widehat{R})=\Phi(\widehat{S} \widehat{R}) .
$$

Рассмотрим некоторую подалгебру $\mathfrak{Q}_{\xi}, \xi \in \Xi$. Не ограничивая общности, мы можем рассматривать элементы этой подалгебры как взаимно коммутирующие линейные самосопряженные операторы в некотором гильбертовом пространстве. В этом случае для любого элемента $\hat{A} \in \mathfrak{Q}_{\xi}$ справедливо спектральное разложение

$$
\Psi(\hat{A})=\int \Psi(\hat{p}(d \lambda)) A(\lambda),
$$

где $\Psi$ - любой ограниченный положительный линейный функционал, а $\hat{p}(d \lambda)$ - проекторы ортогонального разложения единицы. Далее интегралы типа (12) будем записывать в виде

$$
\hat{A}=\int \hat{p}(d \lambda) A(\lambda)
$$

и считать, что все интегралы (и пределы) на алгебре $\mathfrak{A}$ следует понимать в слабой топологии. Соответственно для проекторов $\hat{p}(d \lambda)$ справедливо

$$
\hat{I}=\int \hat{p}(d \lambda) \text {. }
$$

Все элементы подалгебры $\mathfrak{Q}_{\xi}$ имеют единое разложение единицы. Соотношения (12)-(14) чисто алгебраические и не зависят от конкретной реализации элементов алгебры.

Пусть $\hat{p}_{\tau}$ - одномерный проектор. В операторном представлении это проектор на одномерное гильбертово подпространство. Такой проектор обладает свойствами

$$
\hat{p}_{\tau}^{*}=\hat{p}_{\tau}, \quad \hat{p}_{\tau}^{2}=\hat{p}_{\tau}
$$


и не может быть представлен в виде

$$
\hat{p}_{\tau}=\sum_{\alpha} \hat{p}_{\alpha}, \quad \hat{p}_{\tau} \hat{p}_{\alpha}=\hat{p}_{\alpha} \hat{p}_{\tau}=\hat{p}_{\alpha} \neq \hat{p}_{\tau}
$$

Свойства (15) и (16) можно принять в качестве определения одномерного проектора как элемента алгебры.

Рассмотрим некоторый одномерный проектор $\hat{p}_{\tau} \in \mathfrak{Q}_{\xi}$. Учитывая, что (14) - ортогональное разложение единицы, мы можем написать

$$
\hat{p}_{\tau} \hat{p}(d \lambda)=\hat{p}_{\tau} \delta(\lambda-\tau) d \lambda
$$

Пусть $\{\varphi\}_{(\tau)}-$ множество многослойных функционалов, для которых $\varphi_{\xi}\left(\hat{p}_{\tau}\right)=1$. Для всех таких функционалов для каждой наблюдаемой $\hat{A} \in \mathfrak{Q}_{\xi}$ значение $\varphi_{\xi}(\hat{A})$ будет одним и тем же. Действительно, воспользовавшись (13) и (17), получаем

$$
\varphi_{\xi}(\hat{A})=\int A(\lambda)\left[\varphi_{\xi}\left(\hat{p}_{\tau}\right) \delta(\lambda-\tau) d \lambda+\varphi_{\xi}\left(\left(1-\hat{p}_{\tau}\right) \hat{p}(d \lambda)\right)\right]=A(\tau)
$$

Таким образом, множество $\{\varphi\}_{(\tau)}-$ это класс $\varphi_{\xi}$-эквивалентных функционалов. Выделим из него подмножество $\{\varphi\}_{(\xi \tau)}$ многослойных функционалов, стабильных на всех элементах подалгебры $\mathfrak{Q}_{\xi}$. Это подмножество определяет квантовое состояние, которое мы обозначим $\Psi_{\varphi \xi \tau}(\cdot)$ или сокрашенно $\Psi_{\tau}(\cdot)$.

Рассмотрим теперь ГНС-конструкцию, в которой в качестве функционала, порождающего представление, фигурирует $\Psi_{\varphi \xi \tau}(\cdot)$. Пусть $\Phi_{\tau}\left(\hat{p}_{\tau}\right)$ и $\Phi_{\tau}(\hat{I})$ - классы эквивалентности элементов $\hat{p}_{\tau}$ и $\hat{I}$, соответственно. Убедимся, что $\Phi_{\tau}\left(\hat{p}_{\tau}\right)=\Phi_{\tau}(\hat{I})$. Действительно, согласно (8)

$$
\left|\Psi_{\tau}\left(\widehat{R}^{*}\left(\hat{I}-\hat{p}_{\tau}\right)\right)\right|^{2} \leqslant \Psi_{\tau}\left(\widehat{R}^{*} \widehat{R}\right) \Psi_{\tau}\left(\hat{I}-\hat{p}_{\tau}\right),
$$

но $\Psi_{\tau}\left(\hat{I}-\hat{p}_{\tau}\right)=0$, так как $\varphi_{\xi}\left(\hat{p}_{\tau}\right)=1$.

Согласно (10) и (11) для любого $\widehat{S} \in \mathfrak{A}$ имеем

$$
\left(\Phi_{\tau}(\hat{I}), \Pi(\widehat{S}) \Phi_{\tau}(\hat{I})\right)=\Psi_{\tau}(\widehat{S})
$$

Таким образом, значение квантового среднего по квантовому ансамблю $\{\varphi\}_{\xi \tau}$ может быть представлено в виде математического ожидания линейного оператора П $(\widehat{S})$ в состоянии, описываемом вектором $\Phi_{\tau}(\hat{I})=\Phi_{\tau}\left(\hat{p}_{\tau}\right)$ гильбертова пространства. Это позволяет в предлагаемом подходе для вычисления квантовых средних в полной мере использовать математический аппарат стандартной квантовой механики.

Вместе с тем в этом пункте имеется существенное отличие предлагаемого подхода от стандартной квантовой механики. В последней соотношения типа (18) постулируются (постулат Борна). Этот постулат достаточен для квантово-механических расчетов, однако его необходимость не ясна. В противоположность этому в нашем случае равенство (18) является следствием феноменологически необходимых постулатов. 
Убедимся теперь, что функционал, которьй фигурирует в постулате 6 , сушествует. Сначала покажем, что функционал $\Psi_{\varphi \xi \tau}(\cdot)$ должен удовлетворять соотношению

$$
\Psi_{\varphi \xi \tau}(\widehat{S})=\Psi_{\varphi \xi \tau}\left(\hat{p}_{\tau} \widehat{S} \hat{p}_{\tau}\right)
$$

для любого $\widehat{S} \in \mathfrak{A}$. Действительно,

$$
\Psi_{\tau}\left(\hat{p}_{\tau} \widehat{S} \hat{p}_{\tau}\right)=\left(\Phi_{\tau}(\hat{I}), \Pi\left(\hat{p}_{\tau}\right) \Pi(\widehat{S}) \Pi\left(\hat{p}_{\tau}\right) \Phi_{\tau}(\hat{I})\right)=\left(\Phi_{\tau}\left(\hat{p}_{\tau}\right), \Pi(\widehat{S}) \Phi_{\tau}\left(\hat{p}_{\tau}\right)\right)=\Psi_{\tau}(\widehat{S}) .
$$

Теперь докажем следуюшее утверждение.

УТВЕРЖДЕНИЕ. Если $\hat{A} \in \mathfrak{A}_{+}$, mo $\hat{A}_{\tau} \equiv \hat{p}_{\tau} \hat{A} \hat{p}_{\tau}$ uмеет вид $\hat{A}_{\tau}=\hat{p}_{\tau} \Psi_{\tau}(\hat{A})$, где $\Psi_{\tau}(\hat{A})$ - линейный положительный функиионал, удовлетворяющий условию нормировки $\Psi_{\tau}(\hat{I})=1$.

ДокАЗАтЕльство. Будем считать, что элементы $\hat{A}_{\tau}$ и $\hat{p}_{\tau}$ реализованы в виде линейных самосопряженных операторов. Так как $\left[\hat{A}_{\tau}, \hat{p}_{\tau}\right]=0$, то $\hat{A}_{\tau}$ и $\hat{p}_{\tau}$ имеют обшее спектральное разложение единицы. Поскольку проектор $\hat{p}_{\tau}$ одномерньй, то спектральное разложение $\hat{A}_{\tau}$ должно иметь вид $\hat{A}_{\tau}=\hat{p}_{\tau} \Psi_{\tau}(\hat{A})+\left(\hat{I}-\hat{p}_{\tau}\right) \hat{A}_{\tau}$. Поскольку $\hat{p}_{\tau} \hat{A}_{\tau}=$ $\hat{A}_{\tau}$, то отсюда следует

$$
\hat{A}_{\tau}=\hat{p}_{\tau} \Psi_{\tau}(\hat{A})
$$

Из равенства

$$
\hat{p}_{\tau} \Psi_{\tau}(\hat{A}+\widehat{B})=\hat{p}_{\tau}(\hat{A}+\widehat{B}) \hat{p}_{\tau}=\hat{p}_{\tau} \Psi_{\tau}(\hat{A})+\hat{p}_{\tau} \Psi_{\tau}(\widehat{B})
$$

следует линейность $\Psi_{\tau}(\hat{A}+\widehat{B})=\Psi_{\tau}(\hat{A})+\Psi_{\tau}(\widehat{B})$.

По линейности функционал $\Psi_{\tau}(\hat{A})$ может быть расширен на алгебру $\mathfrak{A}: \Psi_{\tau}(\hat{A}+i \widehat{B})=$ $\Psi_{\tau}(\hat{A})+i \Psi_{\tau}(\widehat{B})$, где $\hat{A}, \widehat{B} \in \mathfrak{A}_{+}$.

Положительность функционала следует из соотношения

$$
\Psi_{\tau}\left(\widehat{S}^{*} \widehat{S}\right)=\varphi_{\xi}\left(\hat{p}_{\tau} \Psi_{\tau}\left(\widehat{S}^{*} \widehat{S}\right)\right)=\varphi_{\xi}\left(\hat{p}_{\tau} \widehat{S}^{*} \widehat{S} \hat{p}_{\tau}\right) \geqslant 0 .
$$

Здесь мы воспользовались свойством (2в).

Условие нормировки выполняется в силу равенств

$$
\Psi_{\tau}(\hat{I})=\varphi_{\xi}\left(\hat{p}_{\tau} \Psi_{\tau}(\hat{I})\right)=\varphi_{\xi}\left(\hat{p}_{\tau} \hat{I} \hat{p}_{\tau}\right)=1 .
$$

Таким образом, функционал $\Psi_{\varphi \xi \tau}(\cdot)$, описывающий квантовое среднее, обладает свойством, которое от него требует постулат 6 . Кроме того, он положителен и удовлетворяет условию нормировки. Это как раз те условия, которые должны выполняться для функционала, описываюшего квантовое состояние.

Равенство (20) чисто алгебраическое. Поэтому оно, во-первых, справедливо вне зависимости от конкретной реализации $\hat{A}_{\tau}$ и $\hat{p}_{\tau}$. Во-вторых, значение функционала зависит только от двух факторов: от $\hat{p}_{\tau}$ (квантового состояния) и от $\hat{A}$ как элемента алгебры $\mathfrak{A}$, но не от какой-нибудь частной коммутативной подалгебры ( $\hat{A}$ может принадлежать нескольким таким подалгебрам). Это значит, что функционал $\Psi_{\varphi \xi \tau}(\cdot)$ удовлетворяет постулату 5 .

Функционал, удовлетворяющий соотношению (19), в котором проектор $\hat{p}_{\tau}$ одномерный, соответствует чистому квантовому состоянию. Если проектор $\hat{p}_{\tau}$ был бы многомерным, то состояние было бы смешанным. 


\section{5. ИЛЛЮСТРАЦИЯ}

Применим наши обшие рассуждения к конкретной физической системе. В качестве таковой рассмотрим одномерный гармонический осциллятор. Будем интересоваться функциями Грина для этой системы. Конечно, с помощью ГНС-конструкции можно перейти к стандартной схеме, используюшей гильбертово пространство. Однако можно предложить и более непосредственньй, с точки зрения развиваемого здесь подхода, метод.

Итак, будем считать, что гармонический осциллятор - физическая система, описываемая алгеброй динамических величин $\mathfrak{A}$ - алгеброй с двумя некоммутируюшими эрмитовыми образуюшими $\widehat{Q}$ и $\widehat{P}$, удовлетворяюшими перестановочному соотношению

$$
[\widehat{Q}, \widehat{P}]=i .
$$

Временна́я эволюция в алгебре $\mathfrak{A}$ управляется уравнением

$$
\frac{d \hat{A}(t)}{d t}=i[\widehat{H}, \hat{A}(t)], \quad \hat{A}(0)=\hat{A},
$$

где гамильтониан $\widehat{H}=(1 / 2)\left(\widehat{P}^{2}+\omega^{2} \widehat{Q}^{2}\right)$. Величины $\widehat{Q}, \widehat{P}, \hat{A}, \widehat{H}$ рассматриваются как элементы абстрактной алгебры $\mathfrak{A}$. Элементы $\widehat{Q}, \widehat{P}$ и $\widehat{H}$ неограниченны, поэтому не принадлежат $C^{*}$-алгебре. Однако их спектральные проекторы являются элементами $C^{*}$-алгебры, т.е. $\widehat{Q}, \widehat{P}$ и $\widehat{H}$ - элементы, присоединенные к $C^{*}$-алгебре. Таким образом, в данном случае $\mathfrak{A}$-алгебру следует считать $C^{*}$-алгеброй, дополненной присоединенными элементами.

От эрмитовых элементов $\widehat{Q}$ и $\widehat{P}$ удобно перейти к элементам

$$
\hat{a}^{-}=\frac{1}{\sqrt{2 \omega}}(\omega \widehat{Q}+i \widehat{P}), \quad \hat{a}^{+}=\frac{1}{\sqrt{2 \omega}}(\omega \widehat{Q}-i \widehat{P})
$$

с перестановочным соотношением

$$
\left[\hat{a}^{-}, \hat{a}^{+}\right]=1
$$

и простой временно́й зависимостью

$$
\hat{a}^{-}(t)=\hat{a}^{-} e^{-i \omega t}, \quad \hat{a}^{+}(t)=\hat{a}^{+} e^{+i \omega t} .
$$

Вычислим производяший функционал для функций Грина. В стандартной квантовой механике $n$-временна́я функция Грина определяется формулой

$$
G\left(t_{1}, \ldots, t_{n}\right)=\left\langle 0\left|T\left(\widehat{Q}\left(t_{1}\right) \ldots \widehat{Q}\left(t_{n}\right)\right)\right| 0\right\rangle,
$$

где $T$ - оператор хронологического упорядочивания, $|0\rangle$ - квантовое основное состояние.

Согласно утверждению, доказанному в конце предыдущего раздела, в предлагаемом подходе функция Грина будет определяться формулой

$$
\hat{p}_{0} T\left(\widehat{Q}\left(t_{1}\right) \ldots \widehat{Q}\left(t_{n}\right)\right) \hat{p}_{0}=G\left(t_{1}, \ldots, t_{n}\right) \hat{p}_{0},
$$


где $\hat{p}_{0}$ - спектральный проектор $\widehat{H}$, соответствуюший минимальному значению энергии.

Легко убедиться, что $\hat{p}_{0}$ можно представить в виде

$$
\hat{p}_{0}=\lim _{r \rightarrow \infty} e^{-r \hat{a}^{+} \hat{a}^{-}} .
$$

Как упоминалось ранее, предел следует понимать в смысле слабой топологии $C^{*}$-алгебры.

Сначала докажем вспомогательное утверждение:

$$
\widehat{E}=\lim _{r_{1}, r_{2} \rightarrow \infty} e^{-r_{1} \hat{a}^{+} \hat{a}^{-}}\left(\hat{a}^{+}\right)^{k}\left(\hat{a}^{-}\right)^{l} e^{-r_{2} \hat{a}^{+} \hat{a}^{-}}=0, \quad k, l \geqslant 0, \quad k+l>0 .
$$

Пусть $\Psi$ - ограниченный положительный линейный функционал. Тогда

$$
\Psi(\widehat{E})=\lim _{r_{1}, r_{2} \rightarrow \infty} e^{-r_{1} k-r_{2} l} \Psi\left(\left(\hat{a}^{+}\right)^{k} e^{-r_{1} \hat{a}^{+} \hat{a}^{-}} e^{-r_{2} \hat{a}^{+} \hat{a}^{-}}\left(\hat{a}^{-}\right)^{l}\right) .
$$

Здесь мы воспользовались непрерывностью функционала $\Psi$ и перестановочным соотношением (21). Далее, учитывая, что $\left\|e^{-r \hat{a}^{+} \hat{a}^{-}}\right\| \leqslant 1$, имеем

$$
\begin{aligned}
|\Psi(\widehat{E})| \leqslant & \lim _{r_{1}, r_{2} \rightarrow \infty} e^{-r_{1} k-r_{2} l}\left|\Psi\left(\left(\hat{a}^{+}\right)^{k} e^{-2 r_{1} \hat{a}^{+} \hat{a}^{-}}\left(\hat{a}^{-}\right)^{k}\right)\right|^{1 / 2} \times \\
& \times\left|\Psi\left(\left(\hat{a}^{+}\right)^{l} e^{-2 r_{2} \hat{a}^{+} \hat{a}^{-}}\left(\hat{a}^{-}\right)^{l}\right)\right|^{1 / 2} \leqslant \\
\leqslant & \lim _{r_{1}, r_{2} \rightarrow \infty} e^{-r_{1} k-r_{2} l}\left|\Psi\left(\left(\hat{a}^{+}\right)^{k}\left(\hat{a}^{-}\right)^{k}\right)\right|^{1 / 2}\left|\Psi\left(\left(\hat{a}^{+}\right)^{l}\left(\hat{a}^{-}\right)^{l}\right)\right|^{1 / 2}=0 .
\end{aligned}
$$

Это доказывает равенство (24).

Теперь убедимся в справедливости равенства (23). В терминах элементов $\hat{a}^{+}, \hat{a}^{-}$ гамильтониан $\widehat{H}$ выглядит как $\widehat{H}=\omega\left(\hat{a}^{+} \hat{a}^{-}+1 / 2\right)$. Согласно $(24)$

$$
\lim _{r_{1}, r_{2} \rightarrow \infty} e^{-r_{1} \hat{a}^{+} \hat{a}^{-}} \widehat{H} e^{-r_{2} \hat{a}^{+} \hat{a}^{-}}=\frac{\omega}{2} \lim _{r_{1}, r_{2} \rightarrow \infty} e^{-\left(r_{1}+r_{2}\right) \hat{a}^{+} \hat{a}^{-}} .
$$

Это доказывает равенство (23).

Из формулы (22) следует, что

$$
G\left(t_{1}, \ldots, t_{n}\right) \hat{p}_{0}=\left.\left(\frac{1}{i}\right)^{n} \frac{\delta^{n}}{\delta j\left(t_{1}\right) \ldots \delta j\left(t_{n}\right)} \hat{p}_{0} T \exp \left(i \int_{-\infty}^{\infty} d t j(t) \widehat{Q}(t)\right) \hat{p}_{0}\right|_{j=0} .
$$

По теореме Вика (см. [15])

$$
\begin{aligned}
& T \exp \left(i \int_{-\infty}^{\infty} d t j(t) \widehat{Q}(t)\right)= \\
& \quad=\exp \left(\frac{1}{2 i} \int_{-\infty}^{\infty} d t_{1} d t_{2} \frac{\delta}{\delta \widehat{Q}\left(t_{1}\right)} D^{c}\left(t_{1}-t_{2}\right) \frac{\delta}{\delta \widehat{Q}\left(t_{2}\right)}\right): \exp \left(i \int_{-\infty}^{\infty} d t j(t) \widehat{Q}(t)\right):
\end{aligned}
$$


Здесь : : - операция нормального упорядочивания и

$$
D^{c}\left(t_{1}-t_{2}\right)=\frac{1}{2 \pi} \int d E e^{-i\left(t_{1}-t_{2}\right) E} \frac{1}{\omega^{2}-E^{2}-i 0} .
$$

Выполняя варьирование по $\widehat{Q}$ в правой части (26) и учитывая $(24)$, имеем

$$
\begin{aligned}
& \hat{p}_{0} T \exp \left(i \int_{-\infty}^{\infty} d t j(t) \widehat{Q}(t)\right) \hat{p}_{0}=\exp \left(-\frac{1}{2 i} \int_{-\infty}^{\infty} d t_{1} d t_{2} j\left(t_{1}\right) D^{c}\left(t_{1}-t_{2}\right) j\left(t_{2}\right)\right) \times \\
& \quad \times \hat{p}_{0}: \exp \left(i \int_{-\infty}^{\infty} d t j(t) \widehat{Q}(t)\right): \hat{p}_{0}= \\
&=\hat{p}_{0} \exp \left(-\frac{1}{2 i} \int_{-\infty}^{\infty} d t_{1} d t_{2} j\left(t_{1}\right) D^{c}\left(t_{1}-t_{2}\right) j\left(t_{2}\right)\right)
\end{aligned}
$$

Сравнивая с формулой (25), получаем

$$
G\left(t_{1}, \ldots, t_{n}\right)=\left.\left(\frac{1}{i}\right)^{n} \frac{\delta^{n} Z(j)}{\delta j\left(t_{1}\right) \ldots \delta j\left(t_{n}\right)}\right|_{j=0},
$$

где

$$
Z(j)=\exp \left(\frac{i}{2} \int_{-\infty}^{\infty} d t_{1} d t_{2} j\left(t_{1}\right) D^{c}\left(t_{1}-t_{2}\right) j\left(t_{2}\right)\right)
$$

- производящий функционал.

Как известно, в рамках теории возмушений рассмотрение квантово-полевых систем можно свести к рассмотрению многомерного гармонического осциллятора. Поэтому предложенный метод вычисления производяшего функционала для функций Грина непосредственно обобщается на квантово-полевые модели.

\section{6. ЗАКЛЮЧЕНИЕ}

В настоящей статье была предпринята попытка сформулировать постулаты квантовой механики в максимально слабой форме. Почти все постулаты сформулированы так, что невыполнение любого из них не только нарушает математическую структуру теории, но и приводит к прямому противоречию с экспериментом. Пожалуй, только первый постулат, в котором фигурируют ненаблюдаемые величины, не в полной мере соответствует этому утверждению.

Несмотря на свою слабость, предлагаемые постулаты достаточны для построения математического аппарата, включающего в себя аппарат стандартной квантовой механики. Одновременно с этим в предлагаемом подходе отчетливо видны границы применимости этого аппарата. Его можно применять для описания свойств квантовых ансамблей. Именно в этом случае адекватным понятием является "квантовое состояние". С этим понятием теснейшим образом связан аппарат стандартной квантовой механики.

В противоположность этому в случае одиночного явления адекватным понятием является “физическое состояние”. Это понятие лежит за рамками аппарата стандартной квантовой механики. Его приложение к таким объектам, как “кот Шредингера" или 
Вселенная, не приводит к каким-либо парадоксам. При этом не требуется прибегать к разного рода экзотическим построениям типа суперпозиции живого и мертвого кота или многомировой конструкции Эверетта [16]. Следует отметить, что в предлагаемом подходе полностью отсутствует проблема квантовых парадоксов.

Немаловажной особенностью предложенного подхода является то, что он одинаково приложим как к квантовым, так и к классическим системам, хотя в данной статье почти все внимание было уделено квантовым системам.

Сейчас практически никто из серьезных физиков не пытается свести квантовую физику к классической. Вместе с тем подавляюшее большинство считает, что классическая физика является лишь предельным случаем квантовой, т.е. классическая физика сводится к квантовой. Отсюда возникает стремление проквантовать все встречающиеся в природе поля.

В предлагаемом подходе квантовые и классические поля выступают на равных основаниях, подобно тому, как в теории калибровочных полей выступают абелевы и неаблевы поля. Это открывает новые возможности построения моделей, в которых одновременно присутствуют как квантовые, так и классические поля.

\section{Список литературы}

[1] И. фон Нейман. Математические основы квантовой механики. М.: Наука, 1964.

[2] И. Сигал. Математические проблемы релятивистской физики. М.: Мир, 1968.

[3] А. Эйнштейн, Б. Подольский, Н. Розен. УФН. 1936. Т. 16. С. 440.

[4] E. Schrödinger. Naturwissenschaften. 1935. V. 23. P. 807.

[5] D. Home, M. A. B. Whitaker. Phys. Rep. 1992. V. 210. P. 223.

[6] Д. А. Славнов. ТМФ. 2002. Т. 132. С. 434.

[7] Д. А. Славнов. ТМФ. 2003. Т. 136. С. 437.

[8] У. Рудин. Функциональный анализ. М.: Мир, 1975.

[9] S. Kochen, E.P. Specker. J. Math. Mech. 1967. V. 17. P. 59.

[10] А. Н. Колмогоров. Основные понятия теории вероятностей. 2-е изд. М.: Наука, 1974.

[11] Ж. Неве. Математические основы теории вероятностей. М.: Мир, 1969.

[12] Ю. В. Прохоров, Ю. А. Розанов. Теория вероятностей. Основные понятия. Предельные теоремы. Случайные процессы. Справочник. М.: Наука, 1967.

[13] Ж. Эмх. Алгебраический подход в статистической механике и квантовой теории поля. М.: Мир, 1976.

[14] Ж. Диксмье. $C^{*}$-алгебры и их представления. М.: Наука, 1974.

[15] Н. Н. Боголюбов, Д. В. Ширков. Введение в теорию квантованных полей. М.: Наука, 1984.

[16] H. Everett. Rev. Mod. Phys. 1957. V. 29. P. 454. 